\title{
La mise en fiction d'un voyage insulaire chez Ignacio Aldecoa et Emmanuel Hocquard
}

\author{
José M. OLIVER FRADE \\ Universidad de la Laguna \\ joliver@ull.edu.es
}

Recibido: $14 / 04 / 2016$

Aceptado: 27/09/2016

\section{Résumé}

Les espaces insulaires représentent un sujet récurrent dans la littérature universelle puisqu'ils permettent la coexistence d'une perspective réelle et d'une interprétation allégorique et qu'ils servent de support à un discours dans lequel se confondent le vrai et l'imaginaire. Dans cet article, nous allons mettre en lumière les parallélismes et les divergences qui se produisent dans le traitement symbolico-spatial que deux écrivains contemporains, qui appartiennent à deux domaines linguistiques différents, réservent à un même contexte insulaire. Nous comparerons ainsi les romans Parte de una historia (1967) de Ignacio Aldecoa et Aerea dans les fôrets de Manhattan (1985) de Emmanuel Hocquard, qui se déroulent sur la petite île canarienne de La Graciosa, transformée en la destination d'un voyage existentiel par les deux auteurs.

Mots clés : Littérature de voyages, récit insulaire, Ignacio Aldecoa, Emmanuel Hocquard, Îles Canaries.

\section{La ficcionalización de un viaje insular en Ignacio Aldecoa y Emmanuel Hocquard}

\section{Resumen}

Los espacios insulares son un tema recurrente en la literatura universal, ya que propician la existencia de una perspectiva real junto a una interpretación alegórica, sirviendo así de soporte a un discurso en el que lo imaginario y lo cierto se confunden. En este artículo nos proponemos desentrañar los paralelismos y divergencias que se dan en el tratamiento simbólico-espacial que dos escritores contemporáneos, pertenecientes a dos ámbitos lingüísticos diferentes, llevan a cabo de un mismo escenario isleño. De este modo, comparamos los relatos Parte de una historia (1967) de Ignacio Aldecoa y Aerea dans les fôrets de Manhattan (1985) de Emmanuel Hocquard, ambientados en la pequeña isla canaria de La Graciosa, que ambos narradores convierten en el destino de un viaje existencial.

Palabras clave: Literatura de viajes, relato insular, Ignacio Aldecoa, Emmanuel Hocquard, Canarias. 


\title{
Fictionalizing the Journey to an Island in Ignacio Aldecoa and Emmanuel Hocquard
}

\begin{abstract}
Islands are recurrent in world literature, since they allow for both realistic and allegorical interpretations. As a result, they may support a discourse that blurs the boundaries between the imaginary and the real. In this paper we examine the work of two contemporary writers who belong to two distinctive linguistic cultures. We compare and contrast two novels: Parte de una historia (1967) by Ignacio Aldecoa, and Aerea dans les fôrets de Manhattan (1985) by Emmanuel Hocquard. In both texts the smallest of the Canary Islands, La Graciosa, serves as the destination for an existential journey. Our analysis seeks to tease out the parallelisms and divergences discernible in their symbolic and spatial treatment of the same island context.
\end{abstract}

Key words: Travel literature, island story, Ignacio Aldecoa, Emmanuel Hocquard, Canary Islands.

\section{Referencia normalizada}

Oliver Frade, J. M. (2016). "La mise en fiction d'un voyage insulaire chez Ignacio Aldecoa et Emmanuel Hocquard ». Thélème. Revista Complutense de Estudios Franceses, Vol. 31, Núm. 2 : 315328. http://dx.doi.org/10.5209/rev_THEL.2016.v31.n2.52380

Tout comme le voyage est une question consubstantielle à la propre genèse de la littérature, les îles, associées depuis la nuit des temps aux traversées maritimes, constituent un topos qui a suscité l'intérêt d'un nombre important d'écrivains de différentes cultures.

Ces dernières décennies et en particulier dans l'univers francophone, le motif insulaire a fait l'objet d'un nombre considérable d'études et de rencontres scientifiques multidisciplinaires, qui ont été jusqu'à proposer d'intéressantes perspectives d'approche ( $c f$., parmi d'autres, Fougère, 1995 et 2004 ; Marimotou \& Racault, 1995 ; Trenc, 2001 ; Lestringant, 2002 ; Trabelsi, 2005 ; Racault, 2010 ou Lojacono, 2014). Au-delà du domaine de la théorie littéraire, il faut mentionner les travaux de Joël Bonnemaison $(1997$; 2000) dans le cadre de la géographie ou ceux de la sociologue corse Anne Meistersheim (1997 ; 2001 ; 2006), qui ont dépassé leurs propres champs d'étude pour proposer des visions de la réalité insulaire qui sont à la fois larges et susceptibles d'une interprétation plurielle. C'est ainsi que nous avons estimé très intéressants la distinction que cette spécialiste établit entre les termes « insularité » et « insularisme » et, surtout, ses apports au concept d' « îléité » qu'elle entend comme :

[...] le vécu des insulaires, leur culture, leur imaginaire, tous les comportements induits par la nature particulière de l'espace insulaire, du temps et de la société et qui traverse ainsi et sous-tend tous les phénomènes. L'îléité serait donc cette qualité de la perception et du comportement influencés par la forme spécifique de l'espace insulaire (Meistersheim, 1997 : 110). 
Si Meistersheim oriente ses réflexions dans une perspective plutôt anthropologique et sociologique, la lecture de ses travaux nous invite également à les prendre en compte du point de vue littéraire.

Les recherches que nous menons depuis plus d'une décennie sur l'écriture du voyage aux îles Canaries ${ }^{1}$ nous ont permis de constater, en effet, que les espaces insulaires se sont révélés être un terrain favorable où s'enchevêtrent l'imaginaire et le réel. La charge symbolique que renferme l'archipel canarien, à l'instar de n'importe quel territoire isolé, est accentuée par deux autres traits spécifiques de sa géographie: sa condition d'extrémité occidentale du monde connu jusqu'à la découverte de l'Amérique et les particularités d'une nature singulière marquée par le paysage volcanique et la douceur de son climat. Ceci explique que ces terres ont fait très vite partie de la géographie mythique et que leur représentation littéraire a été largement déterminée - et continue de l'être dans de nombreux textes actuels - par les mythes et les clichés auxquels elles ont été historiquement associées : Îles Fortunées ou des Bienheureux, Jardin des Hespérides, l'Atlantide...2. Il en résulte qu'un nombre considérable d'écrivains y ont fixé leur attention, que ce soit en insistant sur leur charge allégorique ou en se servant de certains aspects de leur histoire et de la réalité canarienne comme toile de fond ou source d'inspiration pour leurs œuvres. Ces deux interprétations du fait insulaire peuvent correspondre - toute proportion gardée - aux notions que nous avons citées plus haut: l'insularité (dimension physique, géospatiale, sociopolitique) et l'îléité (dimension symbolique, perception subjective). Si nous nous limitons aux lettres francophones du siècle dernier, et toujours dans le contexte des îles Canaries, nous trouvons des œuvres de romanciers tels que, par exemple, Jules Verne (L’Agence Thompson \& Co, 1907), Jacques Sadoul (Le sang du dragonnier, 1995), Charlotte Dubreuil (La Restinga, 2000) ou Michel Houellebecq (Lanzarote. Au milieu du monde, 2000) qui illustrent un traitement plus descriptif, tandis que, à l'autre extrémité, d'autres auteurs, comme c'est le cas d'André Breton (Le château étoilé, 1937), Jean Camp (Petite Suite canarienne, 1967), Valentine Penrose («Le carrito», «Le verdino (le chien vert)», 1975), Abdellatif Laâbi («Les îles éternelles », 2000) ou Michel Cosem (plusieurs œuvres, 1994-2010) préfèrent une approche géosymbolique 3 .

\footnotetext{
${ }^{1}$ Nos études sur ce sujet ont été menées dans le cadre de différents projets de recherche depuis 2002. En particulier, cet article a été conçu dans le cadre du projet FFI2011-25994 financé par le Ministère espagnol d'Économie et de la Compétitivité.

${ }^{2}$ L'image symbolique des Canaries et leur relation avec les mythes classiques ont été analysées de façon rigoureuse par Marcos Martínez (2002 et 2009, entre autres).

${ }^{3}$ La représentation des Canaries dans l'œuvre de ces auteurs a été étudiée dans plusieurs travaux au sein du groupe de recherche Fran-Can (cf. le site $<$ http://francan.webs.ull.es $>$ ). Pour une approche géopoétique de ces îles, voir Curell $(2014 ; 2016)$.
} 
Nous allons nous occuper ici de deux écrivains qui combinent ces deux perspectives et qui ont utilisé la petite île de La Graciosa, située à l'un des bouts de l'archipel canarien, comme cadre symbolico-spatial de deux de leurs récits. Nous nous référons à l'Espagnol Ignacio Aldecoa, avec son roman Parte de una historia (Barcelone, Noguer, 1967) ${ }^{4}$, et au Français Emmanuel Hocquard, avec son récit Aerea dans les forêts de Manhattan (Paris, POL, 1985). Tout d'abord, nous consacrerons quelques lignes à la biographie de ces auteurs et à la présentation d'une petite synthèse de ces romans.

Ignacio Aldecoa est né en 1925 à Vitoria et est décédé, de façon soudaine et prématurée, à Madrid à l'âge de 44 ans. Il s'est lancé dans la littérature avec deux livres de poésie, mais c'est sans aucun doute le genre narratif (principalement le conte) qui lui a valu la reconnaissance des écrivains de son époque et de la critique. Faisant partie de ce qu'on appelle la " génération des années 50 » (avec Jesús Fernández Santos, Carmen Martín Gaite, Ana María Matute ou Rafael Sánchez Ferlosio), son écriture, qui est marquée par l'objectivité et le vérisme, s'inscrit dans une espèce de néoréalisme existentialiste qui s'éloigne du message explicitement politique pour faire de la condition humaine l'axe central de son œuvre. Des sujets comme la solitude, la mort, l'incommunication, l'introspection, l'angoisse vitale, l'amour, la fatalité ou la nature sont très présents dans ses récits où l'on apprécie également l'influence de Sartre et de Camus ${ }^{5}$. On soulignera dans sa production littéraire, en plus d'une bonne collection de contes, les romans El fulgor y la sangre (1954), Con el viento solano (1956) et Gran Sol (1957). D'autre part, il faut également mentionner, à cause de sa relation avec les Canaries, son œuvre singulière Cuaderno de godo (1961), un ensemble de treize petits textes qui, en guise de cartes postales, relatent ses impressions et son vécu sur les îles pendant l'hiver 1956-1957.

Parte de una historia est le dernier ouvrage qu'Aldecoa a publié de son vivant et - selon son épouse, la romancière Josefina Rodríguez Aldecoa - un de ceux dont il était le plus satisfait «porque consideraba que era la mejor escrita ( apud Martín Nogales, 1986:4). Le récit est rédigé à la première personne par un voyageur espagnol qui retourne, après quatre ans d'absence, sur une petite île atlantique où il restera quelques jours à observer - plus qu'à partager - la vie quotidienne de ses hôtes, laquelle sera dérangée par l'apparition de trois hommes et d'une femme d'Amérique du Nord que les insulaires ont sauvés après le naufrage de leur yacht. L'irruption de

\footnotetext{
${ }^{4}$ Il existe une traduction française de Jean-Marie Saint-Lu publiée sous le titre de Le naufrage attendu (Nîmes, éditions Jacqueline Chambon, 1997).

${ }^{5}$ Les travaux sur la vie et l'œuvre d'Aldecoa sont très nombreux. Nous renvoyons notamment à Marrero Henríquez (1997), Vadillo Buenfil (2008) et à la compilation d'études coordonnée par Encinar et Valcárcel Rivera (2011).
} 
ces chonis ${ }^{6}$ exhibant des coutumes licencieuses et un état pratiquement permanent d'ivresse est le détonateur qui perturbera l'existence monotone des villageois, marquée par les travaux de la pêche et par des sujets de conversation répétitifs, ainsi que les paisibles vacances d'un couple britannique. Pendant ce temps, le narrateur, qui représente probablement l'auteur dans une stratégie d'autofiction, assiste sans broncher, presque impassible, et parallèlement à l'analyse de sa propre crise vitale, au déroulement des événements qui aboutissent à la fuite vers la mort d'un des Américains. Le roman se termine lorsque les touristes, les naufragés et le visiteur abandonnent l'île quelques jours après cet épisode tragique ${ }^{7}$.

Notre deuxième auteur, Emmanuel Hocquard, un « poète-philologue » comme il se définit lui-même, est né à Cannes en 1940 ; il a passé son enfance à Tanger et a vécu dans différentes villes françaises. Il a été lié très tôt et de différentes manières aux manifestations artistiques de la modernité, à leur divulgation et à leur enseignement, ce qui lui a valu une large reconnaissance dans et à l'extérieur de son pays, surtout en qualité d'éditeur et de traducteur. D'autre part, son côté créatif, aussi bien dans le domaine de la fiction que dans celui de la poésie, est bien apprécié par l'élite littéraire, même si ses œuvres n'ont jamais atteint le grand public ${ }^{8}$. Son écriture se fonde sur une technique minimaliste, qui est tellement descriptive qu'elle en devient parfois photographique, et qui s'alimente aussi bien de l'observation du quotidien que de l'abstraction et de sources érudites ou mythologiques. En particulier dans le domaine de l'écriture poétique, Hocquard s'aligne sur ce qu'on appelle la « modernité négative » qui revendique « une poésie sans accent poétique, aussi sèche qu'une biscotte sans beurre » (Jarrety, 2001). On signalera parmi ses œuvres les plus significatives, en plus du roman Aerea dans les fôrets de Manhattan (prix France Culture de 1985), les récits Un privé à Tanger (1987), La Bibliothèque de Trieste (1988) ou Le consul d'Islande (2000) et les recueils de poèmes Théorie des tables (1992) et Conditions de lumière (2007).

Contrairement à ce qui arrive dans Parte de una historia, dans Aerea dans les forêts de Manhattan, seul le dernier chapitre, intitulé «Alors la couleur...» et qui compte une trentaine de pages, se déroule sur cette île canarienne, qui est appelée ici La Cecilia. La trame de ce roman est axée sur un long voyage que son personnage principal, Adam, un écrivain français sans passé connu, reprend après un bref séjour à New York. Ainsi, une fois terminée l'histoire d'amour qui le retenait aux États-

\footnotetext{
${ }^{6}$ Nom qu'on donne dans ces îles aux étrangers et qui provient d'une corruption phonétique de Johnny (cf. Morera, 2006: 368-369).

${ }^{7}$ Une étude approfondie de ce roman, due à Elide Pittarello, précède l'édition publiée à Madrid chez Castalia en 2005.

${ }^{8}$ Pour plus de détails sur la bio-bibliographie de l'auteur, consulter les ouvrages de Ponsart (2006), Rabaté (2006) ou de Tiberghien (2006). On trouvera également, sur le site web du Centre International de Poésie Marseille, un document contenant une bibliographie exhaustive de cet auteur qui s'étend de 1973 à 2005 (cf. http://www.cipmarseille.com/documents/141_20060307165337.pdf).
} 
Unis et qui l'avait même amené à croire que sa vie errante avait terminé, cet « Ulysse loin des siens » (p. 17) ${ }^{9}$ décide de continuer son odyssée, poursuivi par le souvenir de sa bien-aimée Aerea et sous le regard silencieux du lecteur, son confident et seul compagnon de voyage. Après être passé par les îles grecques et la Turquie, il arrive à Cadix, s'embarque sur un ferry et accoste, par hasard, sur une petite île atlantique. Sa vie sur l'île se déroulera essentiellement autour de la pêche à la ligne et de l'observation, en spectateur privilégié, de la vie quotidienne au café de Don Miguel. Ses journées ennuyeuses seront soudainement interrompues lorsque Juan, son guide et expert pêcheur, est retrouvé noyé. Après cette mort accidentelle, Adam ne parviendra plus à pêcher et remettra en question la raison de sa permanence sur l'île $\mathrm{e}^{10}$.

Nous nous trouvons devant deux romans à mi-chemin entre le récit descriptif et le récit existentiel, contenant une série de représentations de l'insularité et de l'îléité qui offrent certains parallélismes que nous allons essayer de mettre en évidence.

Il faut d'abord signaler, comme c'est d'ailleurs habituel dans les " romans d'île » (cf. Fougère, 2004; Racault, 2010; Cabral \& Santos, 2014), que la trame se déroule dans les deux cas au terme d'un déplacement, dans ce cas une traversée maritime. Une série de détails nous permettent de situer les deux ouvrages dans le domaine de l'écriture du voyage au sens large : le récit se fonde sur un séjour passé à un endroit insolite, la narration suit une chronologie linéaire et est rédigée à la première personne, les éléments descriptifs correspondent au regard posé par le visiteur étranger du haut de son altérité, l'auteur utilise des marqueurs géographiques, sociologiques et linguistiques réels qui apportent de la vraisemblance et de la couleur locale, etc. Ces caractéristiques confirment que les deux auteurs ont une connaissance personnelle du cadre dans lequel se déroulent les romans et qui est sans aucun doute le résultat des séjours qu'ils ont effectués sur l'île. On sait que Ignacio Aldecoa s'est rendu à deux reprises à La Graciosa, son second séjour ayant duré six semaines à l'hiver 19561957. Son penchant pour les îles et son expérience canarienne sont dévoilées par son épouse quelques années plus tard :

Ignacio Aldecoa soñaba, desde su adolescencia, con las islas. Si el mar era su gran pasión, las islas significaban la búsqueda de un sueño literario vivido en las lecturas [...]. En busca de ese sueño, Ignacio descubrió las Islas Canarias. Y aquel descubrimiento le marcó para siempre. Durante seis semanas [a finales de los 50] se refugió en La Graciosa y vivió con sus habitantes una experiencia humana de enorme valor. La autenticidad de los isleños, la cálida hospitalidad que le brindaron, la convivencia en un mundo aislado, generoso, no contaminado, le llevó a escribir la que en su opinión era la mejor de sus novelas: Parte de una historia (J. Aldecoa, 2003: 8-9).

Dans le cas d'Emmanuel Hocquard, il ne nous a pas été possible de nous procurer de données de son séjour au-delà de vagues souvenirs de quelques habitants de l'île.

\footnotetext{
${ }^{9}$ Les citations du récit de Hocquard renvoient à l'édition de 1997 (Paris, POL).
}

${ }^{10}$ Une première approche de ce texte est due à Curell et Oliver (2012). 
Un des traits les plus frappants est le fait que, bien que l'île ne soit désignée dans aucun des deux cas par son véritable nom, les données qui nous sont fournies nous permettent d'identifier, sans le moindre doute, La Graciosa ${ }^{11}$. On constate en effet, dans Parte de una historia, une volonté constante de l'auteur-narrateur d'éviter le nom propre : l'île est simplement appelée «la isla », sans plus ; de façon analogue, Lanzarote, l'île la plus proche et dont elle dépend, est appelée « la Isla Mayor ». Ceci étant, les nombreuses allusions faites aux petits centres de population sous leur nom véritable (Caleta del Sebo, Pedro Barba, Barrio Verde...) ou à certains endroits de la géographie insulaire (Montaña Amarilla, Las Conchas, Los Corrales, Duna Grande, Duna de la Fardela, el Río, etc.) non seulement contredisent cette stratégie de mise en fiction, mais révèlent en fait la véritable identité de ce territoire insulaire : «Las casitas de La Caleta del Sebo, Los Corrales y el Barrio Verde, un desordenado montoncillo de cubos blancos con manchas de colores vivos ; Pedro Barba, una sucia escombrera $[\ldots] \gg($ p. 81$)$.

De son côté, Hocquard préfère utiliser l'hétéronyme de La Cecilia pour désigner l'île, même s'il introduit des toponymes réels, tels que le Río ou la Montaña Clara, ainsi qu'une description précise et fidèle de sa situation géographique qui indiquent clairement qu'il fait référence à La Graciosa :

Située à environ soixante-dix milles marins au large du Sahara, la Cecilia est l'île la plus septentrionale de l'archipel. L'île voisine, dont elle est séparée par un détroit étiré qu'on nomme le Rio, la surplombe de sa falaise... (Hocquard, p. 125).

Les allusions à d'autres aspects de son espace physique contribuent également à renforcer le vérisme de la description de la réalité insulaire. C'est ainsi que les deux auteurs s'accordent, par exemple, à souligner les rues singulières non asphaltées du village $^{12}$ :

De la tienda hemos ido a su casa por las calles de arena con huellas celulares de camellos, huellas tabaleadas de cabras y la grafía aljamiada de las gallinas y las aves de la mar (Aldecoa, p. 10).

Acuña la calle el sol y hay como unas aceras de humedad en la arena que bordea las casas (Aldecoa, p. 24).

Dans les ruelles ensablées, mes espadrilles aux semelles de corde ne font pas de bruit (Hocquard, $p$. 127).

De façon analogue, la description du vent, une constante climatique des archipels atlantiques, présente plusieurs parallélismes :

${ }^{11}$ La Graciosa est l'île la plus grande - avec une extension de 27 kilomètres carrés - et la seule habitée de ce qu'on appelle l'archipel Chinijo, situé au nord de Lanzarote, qui comprend également les îlots d'Alegranza, Montaña Clara et los Roques del Este et del Oeste. Du point de vue administratif, tous dépendent de Teguise, une commune de Lanzarote.

${ }^{12}$ On dit que La Graciosa est la seule île de l'Union européenne qui ne connaît pas l'asphalte. 
El viento sigue soplando, aunque más débilmente. Irrumpe la luna, cárnica y sangrante, recortando los fariones. El son de la mar es un bisbiseo mezclado al hálito del desierto (Aldecoa, p. 218).

Le vent, qui avait soufflé en rafales toute la journée, avait perdu un peu de sa force et le ciel étoilé apparaissait entre les nuages à intervalles irréguliers, révélant alors, sur la crête des vagues, la silhouette de Montagne Claire (Hocquard, p. 152).

Il se peut que le narrateur du roman d'Aldecoa, parce qu'il connait déjà l'île, n'éprouve pas le besoin de souligner d'une façon si prononcée les aspects paysagers comme le fait Adam, pour qui tout est nouveau ou frappant, comme la " petite usine de dessalement d'eau de mer » (Hocquard, p. 126) ou le caractère aride et désolé de ce pays volcanique, qu'il associe à une « étendue désertique des sables et des champs de lave » (Hocquard, p. 126), avec ses dunes et ses roches noires («sable hérissé de pierres et de lapilli », Hocquard, p. 130), en définitive, un « chaos de lave stérile » (Hocquard, p. 134). Les auteurs nous placent ainsi devant un paysage qui, comme nous le verrons plus loin, est en harmonie avec le paysage intérieur des personnages respectifs.

L'ancrage référentiel est renforcé par la présence d'éléments typiques qui apportent de la couleur locale. On constate ce phénomène sur le plan linguistique avec l'emploi de certains mots espagnols dans le cas de Aerea... (pueblo, querido), ou, dans celui de Parte de una historia, avec la prolifération de tournures lexicales, morphosyntaxiques et pragmatiques propres au parler des Canaries ${ }^{13}$. Ces effets sont également obtenus à travers les noms des personnages (qui sont tous des anthroponymes courants aux Canaries), leurs métiers ou leurs particularités, qui, aussi fictifs qu'ils puissent être, s'inspirent de modèles réels. C'est ainsi qu'Aldecoa fait notamment appel à Roque ${ }^{14}$, le propriétaire du magasin et de la pension où loge le visiteur, à Enedina, au Fardelero, à Dominguillo, à Mateo el Guanche, etc. ; tandis que Hocquard utilise Rosita, la fille du propriétaire du bar où se donnent rendez-vous son prétendant Juan, le médecin Don Sebastián et Remedios, auxquels il ajoute des incontournables - compte tenu de l'époque à laquelle le roman a été écrit - guardias civiles qui sont qualifiés par les villageois de « moscas verdes » à cause de la couleur de leurs uniformes.

D'autre part, la pêche - occupation traditionnelle et unique des habitants de l'île est présente tout au long des deux romans et sert également à consolider le réalisme du cadre où se déroule l'action. Les allusions à cet univers sont constantes dans Parte de una historia, comme le montrent les extraits suivants :

${ }^{13}$ Comme, par exemple, l'usage de mots tels que arrequintado, bacinilla, caleo, cachimba, falúa, fardela, jalar, liña, choni, cristiano, maestro... et d'expressions idiomatiques, ainsi que l'emploi surabondant de diminutifs.

${ }^{14}$ Il s'agit probablement de Jorge Toledo, maire de La Graciosa pendant le franquisme. 
Pero él sabe que no hay mejor butaca en el mundo que la suya, que ha debido costar muchas samas, muchos meros y pargos y viejitas y bocinegros y lisas, muchas soldadas de frío a la media noche, calor a mediodía, trabajo y trabajo (Aldecoa, pp. 11-12).

13).

Durante la cena hemos hablado de diferentes cosas relacionadas con el mar y la pesca (Aldecoa, $\mathrm{p}$.

Se acercan las barcas. Los pescadores, fatigados, ateridos, con los mandiles de piel de cabra puestos, hacen las maniobras de atraque (Aldecoa, p. 20).

Al llegar al pueblo nos enteramos que la barca que patronea Maestro Juan ha entrado con una gran calada de arenques. Otras dos barcas han llegado también con mucha pesca. Desde el muelle a las rocas de Los Corrales, las gentes del pueblo transportan, en seras y cajas, la milagrosa pesca (Aldecoa, p. 66).

De façon analogue, le narrateur du roman de Hocquard consacre ses observations et ses réflexions à l'activité halieutique des insulaires, aux espèces les plus communes qui sont capturées ou aux principaux engins utilisés:

À l'heure où le soleil se couche, les barques ressortent dans le Rio pour la pêche aux calmars irisés, alors que sur la place en pente, devant le café de Don Miguel, à la jonction de la jetée et de la plage sèchent les filets couleur de pourpre, les femmes nettoient les poissons du dîner (Hocquard, p. 126127).

À chaque poisson de mer correspond, dirait-on, un poisson d'eau douce. Ainsi, la carpe mélancolique aurait pour demi-sœur marine la vieille aux couleurs vives et aux larges écailles, dont la chair, au goût un peu fade, est appréciée par les insulaires, qui la mangent frite comme la carpe en bien des régions. Pêchée à la ligne dans les eaux de roche, la vieille vorace doit être appâtée aux crustacés ou aux coquillages (Hocquard, p. 138).

Enfin, les deux écrivains coïncident pour décrire l'agora particulière où se retrouvent tous les soirs les pêcheurs et les vieux du coin pour commenter les événements du jour ou pour ressasser de vieilles histoires :

Nado hasta que me enfrío y cuando salgo a la playa de la caleta, donde el cabildo de los viejos espera el crepúsculo comentando y exagerando los escándalos de casa el Fardelero, tiemblo ya (Aldecoa, p. 102).

En el cabildo, ya regresadas algunas barcas, sorprendo tras de las varadas, que exigen ayuda comunal, retazos de conversaciones, soliloquios amargos, pero vuelve el humor, malo o bueno, del trabajo (Aldecoa, p. 213).

Observez les pêcheurs de cette île, alignés chaque soir au coucher du soleil sur ce tronc d'arbre couché au pied d'un mur, qui tient lieu de banc public devant le café de Don Miguel. Leurs yeux vides sont le pathétique reflet du vide de leur esprit au terme d'une existence vouée à la pêche (Hocquard, p. 143-144).

Parallèlement à toutes ces représentations qui évoquent une réalité observée, objective et exacte - ou du moins vraisemblable -, les narrateurs, allant au-delà de la neutralité de la description et se situant sur le plan de la géographie poétique, utilisent quelques-unes des significations ajoutées que renferme l'île en tant que symbole complexe et polysémique. Il est bien connu que l'espace insulaire est considéré 
depuis l'Antiquité comme un territoire magique ${ }^{15}$, « un templum anclado en la inmensidad del mar, habitado por una fuerza positiva o tal vez negativa, un enigma » (Ribera Llopis, 1999 : 21).

Le premier événement qui est raconté et qui finit par devenir un lieu commun, dans une grande partie des textes qui ont abordé la question insulaire au cours de l'histoire de la littérature, est l'arrivée ou le débarquement, qui est la conséquence d'une circonstance violente ou tout au moins non désirée (naufrage, exil, etc.). Dans le roman d'Aldecoa, le visiteur est, dans une certaine mesure, un naufragé, un nomade qui, bien qu'il soit arrivé de façon volontaire sur une île qu'il connaît, ne sait pas vraiment ce qui l'a poussé à s'y rendre ni combien de temps il y restera. Les retrouvailles de ce voyageur avec le territoire occupent la première page du récit et servent à présenter, dans une perspective discursive, le cadre spatial et temporel dans lequel l'intrigue se déroulera :

Ayer, a la caída de la tarde, cuando el gran acantilado es de cinabrio, he vuelto a la isla. [...] Tal vez el pueblo tiene más falúas y se han construido algunas casas, pero he reconocido todo y todo me ha sido familiar después de cuatro años largos, así que he saludado a los amigos como siempre, como si no me hubiera ido [...] (Aldecoa, p. 7).

Quant à lui, le protagoniste du roman de Hocquard est décrit comme un véritable naufragé, "parti un bel après-midi de Cadix », qui ne s'explique pas « ce qui peut amener un homme à s'embarquer un beau jour dans le but d'aller pêcher loin de chez lui » (Hocquard, p. 144). Se reconnaissant « en fuite et sans port d'attache », il finira par se sentir « en quelque sorte vomi par le vaisseau blanc, après deux jours et deux nuits de voyage, sur la côte sauvage de la Cecilia » (Hocquard, p. 144).

Le caractère d'univers clos, inconnu et, en général, lointain constitue un autre attribut symbolique des espaces insulaires et représente une constante dans leur caractérisation en territoire mythique. Dans ce cas, la double insularité qui découle du fait d'être une île de très petite dimension au sein d'un archipel contribue encore davantage à renforcer cette dimension allégorique. Ainsi, Adam se sent face à « un monde coupé du monde dont il n'offre plus guère qu'un écho affaibli et lointain » (Hocquard, p. 128), tandis que le narrateur anonyme du roman d'Aldecoa exprime de la façon suivante l'énorme distance, et physique et mentale, qui le sépare de ses racines :

Ahora rememoro, encontrando una suerte de compasión gozosa, todo lo que ha sido encastillado desastre y orgulloso cansancio de mí mismo. ¿Hasta dónde el orgullo puede desarraigarnos? Ahora rememoro, estando a muchas millas de mar, a muchos quilómetrosde mi tierra, la ciudad de desasosiego que he abandonado. Aquí, en esta isla y en esta mañana bruñida, comienzo a comprenderme distanciado de la imagen que tengo de mía, allá, lejos, como en una historia sucedida a otro (Aldecoa, p. 64).

\footnotetext{
${ }^{15}$ Pour plus de détails sur le concept d' « île mythique » et sur sa présence dans la littérature grécolatine, nous renvoyons à Marcos Martínez (1997).
} 
Ce sens principal a donné lieu à une série de valeurs, au contenu négatif et aussi traditionnellement associées à l'îléité, telles que l'isolement, la solitude ou la mort ${ }^{16}$ :

Arena, falta de agua, vegetación de desierto, incomunicación, soledad de supervivientes (Aldecoa, p. 95).

Y yo siento un extraño vacío dentro de mí, como si - no hay razón alguna para confirmarlo - la soledad temida de otras veces ya me hubiese empapado y se hubiera evaporado muy repentinamente (Aldecoa, p. 104).

[...] ce monde en déclin (Hocquard, p. 129).

[...] le paysage mort qu'il avait sous les yeux (Hocquard, p. 144).

[...] le grand corps momifié de l'île [soudé] à son sarcophage (Hocquard, p. 153).

Dans une apparente contradiction avec ce qui précède et comme conséquence de l'ambivalence propre à la symbologie insulaire - « dans l'île tout se change en son contraire » (Meistersheim, $1997: 112$ ) - l'île se présente également comme une terre d'accueil, comme un refuge qui, dans Parte de una historia, permet aussi bien à des touristes britanniques de passer leurs vacances intimes dans un cadre édénique qu'à quatre riches Américains de se sauver d'un naufrage. Quant aux personnages principaux, l'île est l'endroit où ils trouvent abri au terme de la fuite qu'ils ont entreprise et où ils essaient d'obtenir une réponse aux problèmes et aux angoisses qu'ils croient avoir laissés derrière eux. Le narrateur du roman d'Aldecoa décrit ce sentiment de la manière suivante :

Estoy lejos, en mi propio naufragio, siendo una presencia ajena, alguien que no puede compartir lo que sucede, alguien que no deja compartir lo que pasa. Todos mis riesgos están fuera de esta isla, y aquí en este regazo es donde se desencadenan otros riesgos que no me alcanzan (Aldecoa, pp. 41-42).

Ce dilemme est posé à plusieurs reprises à travers différents soliloques :

Estoy otra vez en la isla y de huida. ¿De quién huyo? No sabría decírmelo. Todo es demasiado vago. ¿Tengo alguna razón? ¿Por qué y de qué? No, no sabría decírmelo. ¿Y estoy aquí porque es aquí donde puedo encontar algo? No sabría decírmelo. Huir acaso explica la huida. Y estoy aquí junto a esta barca, solo en la noche. ¿Y, estoy como esta barca, rumbo al vacío y para siempre? (Aldecoa, p. 35).

- ¿Y si me quedo para siempre? -pregunto, intrigándole (Aldecoa, p. 205).

Adam, de son côté, tel le sophiste de Platon, essaie de trouver une réponse à ses inquiétudes vitales dans la pratique de la pêche à la ligne. C'est ainsi qu'il affronte la pêche en solitaire, jour après jour, en attendant patiemment la secousse qui lui indiquera qu'un poisson a mordu ; et pendant ce bref laps de temps, fugace signe de vie provenant de l'océan, «le pêcheur tient dans sa main, par la grâce d'un invisible

${ }^{16}$ Il est curieux d'observer, comme l'indique François Moureau (1989: 7-8), que le profond symbolisme létal qui est associé à l'île contraste avec son immortalité dans sa condition de substance mythique. 
poisson, son passé et son avenir » (Hocquard, p. 147) ; en définitive, toute une possibilité de vie : « [...] entre tes mains, Adam, tiens ferme le fil qui relie ta vie, pardelà la remuante surface des flots, à une autre vie encore inconnue de toi. Tiens le fil de la vie et ne t'endors pas sous le soleil : une lame imprévisible pourrait bien t'emporter [...] » (Hocquard, p. 141).

D'autre part, dans un des monologues manifestement adressés au lecteur, Adam essaie d'expliquer - et de s'expliquer à lui-même - les véritables raisons qui ont pu le pousser à entreprendre une traversée maritime pour aboutir sur cette petite île : "Quel mobile peut pousser un homme qui a beaucoup lu et qui a réfléchi à s'embarquer un soir d'été sur un grand bateau blanc et à parcourir des milles et des milles sur l'océan afin d'aller pêcher à la ligne, tout seul face à l'horizon vide, sur un rivage désolé ? » (Hocquard, p. 143).

En effet, La Cecilia ne constitue pas une halte quelconque dans le périple à travers le monde de notre héros, mais semble être une dernière escale, un port sûr, propice à la connaissance de soi, où il pourra peut-être trouver, enfin, la stabilité émotionnelle qui lui permettra de reprendre son voyage ou de retourner au point de départ: « Et qu'une fois échoué en ce lieu [...], il ait alors pensé découvrir, dans le miroir enfoui de ce qui vit sous la mer, de quoi ranimer en lui une étincelle de vie » (Hocquard, p. 144).

La mort tragique d'un des personnages secondaires constitue, dans les deux récits, un point d'inflexion qui détermine la fin du séjour insulaire des protagonistesnarrateurs, que ce soit parce qu'ils y ont trouvé la réponse à leur quête, parce qu'ils ont réalisé qu'elle n'était pas là ou parce qu'ils ont reconsidéré leur situation. Au bout du compte, dans les deux ouvrages le lecteur pressentait depuis le début que la fonction de l'île serait limitée : dans celui d'Aldecoa, son propre titre nous laisse entendre qu'il s'agit d'une "partie d'une histoire», d'un épisode dont nous ne connaissons ni le passé ni l'avenir et qui se termine de façon ouverte ${ }^{17}$; dans Aerea dans les forêts de Manhattan, les faits situés à La Cecilia constituent le dernier chapitre d'une série d'étapes d'un voyage physique et mental qui débouche, en formant une structure circulaire, sur un retour au début du roman.

En tout état de cause, il ne fait aucun doute que Ignacio Aldecoa et Emmanuel Hocquard attribuent à la petite île de La Graciosa une fonction qui va bien au-delà du décor : ils en font un élément structurel, un protagoniste de leur histoire qui intervient directement dans la trame, qui conditionne le reste des personnages et qui devient l'alter ego des narrateurs.

17 En effet, Aldecoa lui-même affirmait dans une interview avec José Julio Perlado (1967 : 21) que le roman est ouvert à l'avant et à l'arrière. 


\section{RÉFÉRENCES BIBLIOGRAPHIQUES}

Aldecoa, I., (1967) Parte de una historia. Madrid, Alfaguara.

Aldecoa, J., (2003) «Prólogo », in Aldecoa, I., Cuaderno de godo. Santa Cruz de Tenerife, Ediciones Idea.

Bonnemaison, J., (1997) Les fondements géographiques d'une identité. L'archipel du Vanuatu, essai de géographie culturelle. Paris, Editions de l'ORSTOM Bonnemaison, J., (2000) La géographie culturelle. Paris, Éditions du CTHS. Cabral, M. J. \& A. C. Santos (éds.), (2014) Les possibilités d'une île. Paris, Pétra.

Curell, C., (2014) « La géosymbolique des îles: les Canaries dans la poésie française actuelle », in Moniz, A. I., Faria, D., Martins Coelho, L. \& J. D. de Almeida (coords.), L'île: images, imaginaire et fiction [En ligne]. Porto, Universidade do Porto, pp. 59-72. Disponible sur: http://ler.letras.up.pt/uploads/ficheiros/12586.pdf. [Dernier accès le 1/04/2016].

Curell, C., (2016, prévu): « Diario poético de un viaje: Jean Camp en Canarias », in Aragón Ronsano, F. \& J. A. López Sánchez. (éds.), Historias de viajes. Berne, Peter Lang.

Curell, C. \& J. M. Oliver, (2012) « La isla de la Graciosa, última escala de un viaje narrativo », in Curell, C., G. de Uriarte, C. \& J. M. Oliver (coords.), Estudios franceses en homenaje a Berta Pico. La Laguna, Servicio de Publicaciones de la Universidad de La Laguna, pp. 111-119.

Encinar, Á. \& C. Valcárcel Rivera (coords.), (2011) Ignacio Aldecoa, maestro del cuento : nuevas perspectivas sobre su obra y Antología de cuentos. Madrid, Edaf.

Fougère, É., (1995) Les voyages et l'ancrage. Représentation de l'espace insulaire à l'Age classique et aux Lumières (1615-1797). Paris, L'Harmattan.

Fougère, É., (2004) Escales en littérature insulaire. Îles et balises. Paris, L'Harmattan.

Hocquard, E., ([1985] 1997) Aerea dans les forêts de Manhattan. Paris, POL.

Jarrety, M. (dir.), (2001) Dictionnaire de poésie de Baudelaire à nos jours. Paris, PUF.

Lestringant, F., (2002) Le Livre des îles : atlas et récits insulaires de la Genèse à Jules Verne. Genève, Librairie Droz.

Lojacono, F., (2014) Roman de l'île et robinsonnade ontologique. Paris, Éditions Pétra.

Marimotou, J.-C. \& J.-M. Racault (coord.), (1995) L'insularité thématique et représentations. Paris, L'Harmattan.

Marrero Henríquez, J. M., (1997) Documentación y lirismo en la narrativa de Ignacio Aldecoa. Las Palmas de Gran Canaria, Servicio de Publicaciones de la Universidad de Las Palmas de Gran Canaria.

Martín Nogales, J. L., (1986) Los cuentos de Ignacio Aldecoa. Madrid, Cátedra.

Martínez, M., (1997) « Islas míticas », in Díez de Velasco, F., Martínez, M. \& A. Tejera (éds.), Realidad y mito. Madrid, Ediciones Clásicas, pp. 19-43.

Martínez, M., (2002) Las Islas Canarias en la antigüedad clásica: mito, historia e imaginario. La Laguna, Centro de la Cultura Popular Canaria. 
Martínez, M., (2009) « Las Canarias en el mar de los mitos », in Revista de Occidente. $\mathrm{N}^{\circ} 342$, pp. 83-108.

Meistersheim, A., (1997) « Figures de l'îléité, image de la complexité... », in Reig, D. (comp.), Île des merveilles. Mirage, miroir, mythe. Paris, L'Harmattan, pp. 109-124.

Meistersheim, A., (2001) Figures de l'île. Ajaccio, DCL éditions.

Meistersheim, A., (2006) "Insularité, insularisme, îléité, quelques concepts opératoires », in Les îles malgré l’Europe. Bastia, Materia Scritta, coll. "Les cahiers de l'IDIM », pp. 159-179.

Morera, M., (2006) Diccionario histórico-etimológico del habla canaria. Puerto del Rosario, Servicio de Publicaciones del Cabildo de Fuerteventura.

Moureau, F., (1989) L'île, territoire mythique. Paris, Aux amateurs des livres.

Perlado, J. J., (1967) « Entrevista con Ignacio Aldecoa », in El Alcázar, 3 mai, p. 21.

Ponsart, E., (2006) Bibliographie d'Emmanuel Hocquard, 1973-2005. Marseille, Centre International de Poésie Marseille.

Rabaté, D., (2006) Le Chaudron fêlé. Écarts de la littérature. Paris, Seghers.

Racault, J.-M., (2010) Robinson et compagnie: aspects de l'insularité politique de Thomas More à Michel Tournier. Paris, Petra.

Ribera Llopis, J. M., (1999) « Si miràveu més luny, veuríeu una isla..., sugiere Ovidio en lengua de Francesc Alegre: hombres que miran y parajes mirados en la literatura medieval », in Cuadernos del CEMyR. Vol. 7, pp. 11-24.

Tiberghien, G., (2006) Emmanuel Hocquard. Paris, Seghers.

Trabelsi, M., (2005) L'insularité, Clermont-Ferrand, Presses universitaires Blaise Pascal.

Trenc. E. (coord.), (2001) Au bout du voyage. L'île, mythe et réalité. Reims, Presses Universitaires de Reims.

Vadillo Buenfil, C., (2008) «La intertextualidad existencial entre Parte de una historia de Ignacio Aldecoa y algunas narraciones de Albert Camus », in Hesperia. Anuario de filología hispánica. Vol. XI, nº 1, pp. 87-98. 